\title{
Twenty-Six Moves Suffice for Rubik's Cube
}

\author{
Daniel Kunkle \\ College of Computer Science \\ Northeastern University \\ Boston, MA 02115 / USA \\ kunkle@ccs.neu.edu
}

\author{
Gene Cooperman \\ College of Computer Science \\ Northeastern University \\ Boston, MA 02115 / USA \\ gene@ccs.neu.edu
}

\begin{abstract}
The number of moves required to solve any state of Rubik's cube has been a matter of long-standing conjecture for over 25 years - since Rubik's cube appeared. This number is sometimes called "God's number". An upper bound of 29 (in the face-turn metric) was produced in the early 1990's, followed by an upper bound of 27 in 2006.

An improved upper bound of 26 is produced using $8000 \mathrm{CPU}$ hours. One key to this result is a new, fast multiplication in the mathematical group of Rubik's cube. Another key is efficient outof-core (disk-based) parallel computation using terabytes of disk storage. One can use the precomputed data structures to produce such solutions for a specific Rubik's cube position in a fraction of a second. Work in progress will use the new "brute-forcing" technique to further reduce the bound.
\end{abstract}

Categories and Subject Descriptors: I.1.2 [Symbolic and Algebraic Manipulation]: Algebraic algorithms

General Terms: Algorithms, Experimentation

Keywords: Rubik's cube, upper bound, permutation groups, fast multiplication, disk-based methods

\section{INTRODUCTION}

Over the decades, short solutions to Rubik's cube have provided a fascination - both for researchers in techniques of search and enumeration and for hobbyists. For researchers, Rubik's cube serves as a well-known challenge problem against which otherwise diverse methods can be compared. In 1982, Singmaster and Frey [2] ended their book on Cubik Math with the conjecture that "God's number" is in the low 20's.

No one knows how many moves would be needed for "God's Algorithm" assuming he always used the fewest moves required to restore the cube. It has been proven that some patterns must exist that require at least seventeen moves to restore but no one knows what those patterns may be. Experienced group theorists have

\footnotetext{
${ }^{*}$ This work was partially supported by the National Science Foundation under Grants ACIR-0342555 and CNS-06-19616.
}

Permission to make digital or hard copies of all or part of this work for personal or classroom use is granted without fee provided that copies are not made or distributed for profi t or commercial advantage and that copies bear this notice and the full citation on the fir rst page. To copy otherwise, to republish, to post on servers or to redistribute to lists, requires prior specifi $\mathrm{c}$ permission and/or a fee.

ISSAC'07, July 29-August 1, 2007, Waterloo, Ontario, Canada.

Copyright 2007 ACM 978-1-59593-743-8/07/0007 ...\$5.00 conjectured that the smallest number of moves which would be sufficient to restore any scrambled pattern that is, the number of moves required for "God's Algorithm" - is probably in the low twenties.

This conjecture remains unproven today. At the time of this conjecture, the best known bounds were a lower bound of 17 and an upper bound of 52 [2]. The current best lower bound is 20 [7]. Before this work, the best known upper bound was 27 [5]. Here, we improve that bound to 26 .

Note that in all cases, we consider a move to be any quarter or half turn of a face of the cube, also known as the face-turn metric. We do not consider the alternative quarter-turn metric, which defines a half-turn to be two moves.

We present a new, algebraic approach that concentrates on analysis of cosets for the corresponding mathematical group. The novelty is based on a combination of:

- a subgroup chain of length two based on the square subgroup (order 663,552)

- a new fast multiplication (requiring less than 100 nanoseconds) of either a symmetrized coset or a symmetrized group element by a generator (see Sections 3, 4.1 and 5 for definitions);

- efficient parallel computation using the aggregate bandwidth of parallel disks for $7 \mathrm{~TB}$ of intermediate storage. (The aggregate bandwidth is comparable to the bandwidth of a single RAM subsystem.)

- an efficiently computable perfect hash function of the set of symmetrized cosets (see Section 4.1 for definitions), and efficient computation of its inverse; and

- a compact representation of the coset graph data structure using four bits per state to encode $1.4 \times 10^{12}$ states.

The foundation of the method is to determine the maximal distance from the identity in both the square subgroup and the corresponding coset graph. At heart, the computation is simply breadthfirst search using the 18 generators of Rubik's cube (including squares and inverses of generators). The out-of-core computation is required for the construction of the coset graph involving over 65 trillion cosets.

The problem is reduced in space and time through the use of the 48 symmetries of Rubik's cube (generated by the 24 symmetries of a geometric cube, plus a geometric inversion). These symmetries are generator-preserving automorphisms in their action on the group of Rubik's cube. We can define both equivalence classes of group elements (symmetrized group elements) and equivalence classes of cosets (symmetrized cosets) under the automorphism. This reduces the size of the coset graph to approximately 1.4 trillion symmetrized cosets. 
The paper is organized as follows. Section 2 briefly reviews some related work. Section 3 presents the overall algorithm from a high level. Section 4 presents background and some basic concepts. In particular, this includes the definitions of symmetrized group element and symmetrized coset. Section 5 describes the fast multiplication algorithm, along with the perfect hash function. Section 6 presents the details of finding a relatively tight upper bound on the distance to the identity element of all elements of a symmetrized coset. Section 7 presents details on the computations and final results.

\section{RELATED WORK}

One approach to finding bounds on solutions to Rubik's cube would be to produce the entire Cayley graph for the corresponding group. Cooperman, Finkelstein and Sarawagi used this method to show that 11 moves suffice for Rubik's $2 \times 2 \times 2$ cube [1]. For the full $3 \times 3 \times 3$ Rubik's cube, this is not feasible, since it has over $4.3 \times 10^{19}$ states.

The first published upper bound was 52. Discovered by Thistlethwaite [2], it was based on solving the cube in a series of four steps, corresponding to a chain of subgroups of length four. The four steps were proven to have worst case lengths of $7,13,15$, and 17, for the total of 52 .

In 1992, this algorithm was improved by Kociemba [3] to use a subgroup chain of length two. In 1995, Reid [6] proved the worst case for the two steps was 12 and 18, for a total upper bound of 30. Further analysis showed that the worst case never occurs, and so a bound of 29 was shown. This bound was further refined by Radu [5] in 2006 to 27, which was the best upper bound before this work.

Besides work into methods with provable worst cases, several optimal solvers with no worst case analysis have been developed. The method developed by Kociemba and analyzed by Reid has a natural extension that guarantees optimal solutions. Korf [4] used similar techniques to optimally solve ten random cube states, one in 16 moves, three in 17 moves, and the remaining six in 18 moves.

\section{OVERVIEW OF APPROACH}

The group of Rubik's cube is decomposed into a chain of length two, using the square subgroup (the subgroup generated by using only half-turns of the faces) as the intermediate subgroup. The square subgroup has only 663,552 elements, and there are approximately $6.5 \times 10^{13}$ cosets in Rubik's group. This is in contrast to previous related approaches, which used subgroups that provided subproblems of nearly equal size.

The overall strategy for an improved upper bound for Rubik's Cube has the following three phases. First, in Section 3.1, we produce a graph of the symmetrized subgroup (symmetrized Cayley graph), with subgroup elements as nodes and the generators as edges, and show that no element has a distance of more than 13 from the identity. Second, In Section 3.2, we produce a graph of the symmetrized cosets (symmetrized Schreier coset graph), and show that no coset has a distance more than 16 from the trivial coset. These first two steps provide an initial upper bound of 29. Finally, in Section 6, we efficiently find still tighter bounds, by examining the distance from group elements in the symmetrized coset to the trivial group element, yielding the final upper bound of 26 .

\subsection{Construction of symmetrized Cayley sub- group graph}

Because of the small size of the square subgroup, only 15,752 after reduction by symmetries, these computations are negligible when compared to the corresponding computations for the cosets.

First, we constructed the Cayley graph of the square subgroup by breadth-first search, using the square generators. This computations took only seconds, even using a simple implementation on a single computer. Then, we found the optimal solution for all elements of the square subgroup, where we allowed any of the 18 generators to be used. This was done using a bidirectional search for each of the subgroup elements, using a day of CPU time.

We chose to use bidirectional search for this case, which proceeds in two phases. First, we performed a forward search to depth 7 using all of the generators. Then, we performed one backward search for each of the 15,752 elements of the square subgroup. These backwards searches required anywhere from milliseconds to a few hours in the worst case. Overall, this optimization took less than one day, requiring no parallelization.

Table 1 in Section 7 shows the distribution of elements for the square subgroup, both for just the square generators and when allowing all generators of the cube group.

\subsection{Construction of symmetrized Schreier coset graph}

The construction is essentially a queue-based implementation of breadth-first search. The complexity in the algorithm is primarily due to the necessity of reducing the search space by the 48 symmetries of the cube, and of using disk.

The primary data structure is an almost perfectly dense hash array of $1.5 \times 10^{12}$ entries, corresponding the the range of hash indices for all symmetrized cosets. The entries hold a four bit value describing the level at which the corresponding symmetrized coset occurs, for a total array size of $685 \mathrm{~GB}$.

Algorithm 1 describes how this array is filled with the level at which every symmetrized coset occurs in the breadth-first search. It uses an iterative process with two phases, generating and merging, with one such iteration per level. As an optimization, the breadthfirst search is initially conducted using only main memory, as in the traditional case, and switches to the parallel disk-based version once RAM limitations are met (in this case, at level 9).

\section{NOTATION AND BASIC CONCEPTS}

\subsection{Group theory definitions}

We review the formal mathematical definitions. Recall that a group $G$ is a set with multiplication and an identity $e(e g=$ $g e=g)$, inverse $\left(g g^{-1}=g^{-1} g=e\right)$, and an associative law $((g h) k=g(h k))$. A permutation of a set $\Omega$ is a one-to-one and onto mapping from $\Omega$ to $\Omega$. Composition of mappings provides the group multiplication, and the group inverse is the inverse mapping. A permutation group $G$ is a subset of the permutations of a set $\Omega$ with the above operations. A subgroup $H<G$ is a subset $H$ that is closed under group operations. A group $G$ has generators $S \subseteq G$, written $G=\langle S\rangle$, if any element of $G$ can be written as a product of elements of $S$ and their inverses. The order of the group is the number of elements in it, $|G|$.

Given a group $G$ and a subgroup $H<G$, a coset of $H$ is the set $H g=\{h g: h \in H\}$. A subgroup $H<G$ partitions the group into cosets. The set of all cosets is written $G / H$. The conjugate of $g$ by $h$ is defined by $h^{g} \stackrel{\text { def }}{=} g^{-1} h g . N<G$ is normal in $G$ if $\forall n \in N, g \in G, n^{g} \in N$.

An automorphism $\alpha$ of a group $G$ is a one-to-one and onto mapping of $G$ such that for $g_{1}, g_{2} \in G$, one has $\alpha\left(g_{1} g_{2}\right)=\alpha\left(g_{1}\right) \alpha\left(g_{2}\right)$ The informal idea of symmetries of Rubik's cube has its formal analogue in automorphisms.

A Cayley graph of a group $G$ with generators $S$ is a directed graph whose vertices are the elements of $G$ and whose directed edges, $\left(g_{1}, g_{2}\right)$, satisfy $g_{1} s=g_{2}$ for some edge label $s \in S$. Since our chosen generating set for Rubik's cube is preserved under inverses, the Cayley graph of Rubik's cube can also be considered an undirected graph. A Schreier coset graph of a group $G$ with generators $S$ and subgroup $H<G$ is a graph whose vertices are the elements of $\mathrm{G} / \mathrm{H}$ and whose edges, $\left(\mathrm{Hg}_{1}, \mathrm{Hg}_{2}\right)$, satisfy 


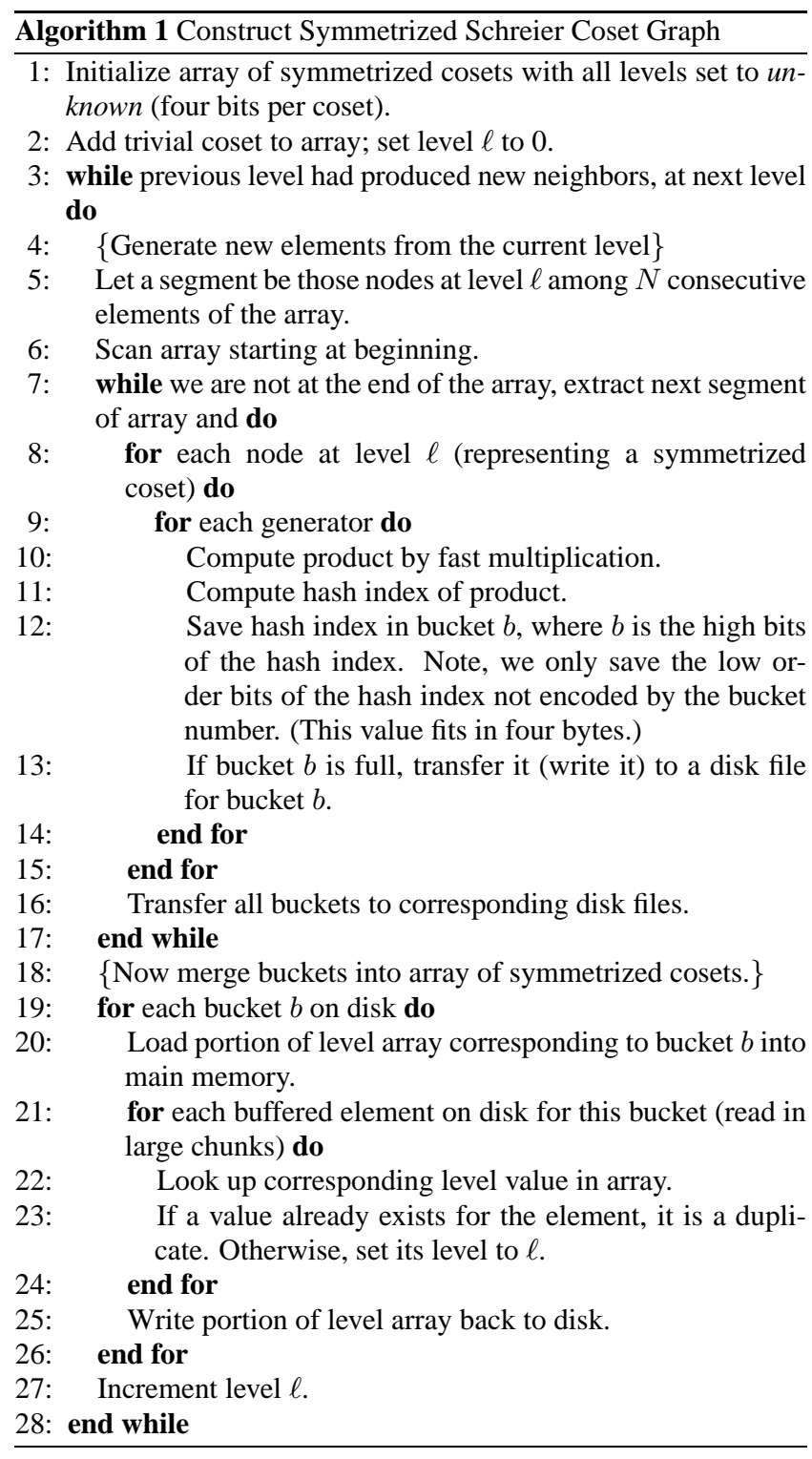

$\mathrm{Hg}_{1} s=\mathrm{Hg}_{2}$ for some edge label $s \in S$.

Symmetrized group elements and symmetrized cosets.

While the above definitions are standard, we extend them to symmetrized group elements and symmetrized cosets. Let $A$ be a group of automorphisms of a group $G$. A symmetrized group element $g^{A}$ for $g \in G$ is the set $\{\alpha(g): \alpha \in A\}$.

Given a subgroup $H<G$, let $A$ be a group of automorphisms of $G$ that also preserve $H(\forall h \in H, \alpha \in A, \alpha(h) \in H)$. The symmetrized coset $\mathrm{Hg}^{A}$ is the set of elements $\{h \alpha(g): h \in H, \alpha \in$ $A\}=\cup_{\alpha \in A} H \alpha(g)$. (Note $\alpha\left(H g^{A}\right)=H g^{A} \forall \alpha \in A$.)

Let $A$ be a group of automorphisms of $G$. Assume that $A$ preserves the generating set $S$ of $G$. Then the symmetrized Cayley graph for $(G, S, A)$ is the directed graph with vertices $\left\{g^{A}: g \in\right.$ $G\}$ and with edges $\left(g_{1}^{A}, g_{2}^{A}\right)$ satisfying for some edge label $s \in S$ : $\forall g_{1}^{\prime} \in g_{1}^{A}, g_{1}^{\prime} s \in g_{2}^{A}$.

Without loss of generality, it suffices to consider only edge labels satisfying $g_{1} s \in g_{2}^{A}$ for a distinguished group element $g_{1} \in g_{1}^{A}$. Note that the edge label in a symmetrized Cayley graph is not unique since we could equally well consider $\alpha\left(g_{1}^{\prime}\right) \alpha(s) \in \alpha\left(g_{2}\right)$ as defining that edge. Hence, the edge $\left(g_{1}^{A}, g_{2}^{A}\right)$ has edge label $s$ such that $g_{1} s=g_{2}$ and also edge label $s^{\prime}=\alpha(s)$ for some $\alpha \in A$, such that $\alpha\left(g_{1}\right) \alpha(s)=\alpha\left(g_{2}\right)$. For any element $g_{1}^{\prime} s \in g_{2}^{A}$ satisfying $g_{1}^{\prime} \in g_{1}^{A}$, there is an $\alpha \in A$ with $\alpha\left(g_{1}^{\prime}\right)=g_{1}$ and so the edge $\left(\left(g_{1}^{\prime}\right)^{A},\left(g_{1}^{\prime} s\right)^{A}\right)=\left(g_{1}^{A},\left(g_{1} \alpha(s)\right)^{A}\right)$.

Similarly, assuming that $G=\langle S\rangle, H<G$, and $A$ preserves $S$, one defines the symmetrized Schreier coset graph for $(G, H, S, A)$ as the directed graph with vertices $\left\{H g^{A}: g \in G\right\}$ and with edges $\left(g_{1}^{A}, g_{2}^{A}\right)$ satisfying $H g_{1}^{\prime} \in H g_{1}^{A}$ and $H g_{1} s \in H g_{2}^{A}$. As before, in order to find all neighbors of $g_{1}^{A}$ in a symmetrized Cayley graph, it suffices to choose any distinguished element $g^{\prime} \in H g_{1}^{A}$, and the set of neighbors is

$$
\left\{\left(H\left(g^{\prime}\right)^{A}, H\left(g^{\prime} s\right)^{A}\right): s \in S, g^{\prime} s \notin H\left(g^{\prime}\right)^{A}\right\} .
$$

Note that for identity $e \in G$, the trivial coset $H e=H$ and the trivial symmetrized coset $H e^{A}=H$ are equal. For our work, we require the following property:

Let $g^{\prime} \in H g^{A}$. Then the distance from the symmetrized coset $\mathrm{Hg}^{A}$ to the trivial symmetrized coset $H$ in the symmetrized Schreier coset graph is the same as the distance from the coset $\mathrm{Hg}^{\prime}$ to the trivial coset $H$ in the Schreier coset graph.

The property is easy to prove. If there is a word $w^{\prime}$ with distinguished element $g^{\prime \prime} \in H g^{A}$ such that $g^{\prime \prime} w^{\prime} \in H e^{A}=H$, then there is an $\alpha \in A$ with $\alpha\left(g^{\prime \prime}\right)=g^{\prime}$, and therefore $g^{\prime} \alpha\left(w^{\prime}\right)=$ $\alpha\left(g^{\prime \prime}\right) \alpha\left(w^{\prime}\right) \in H$. Since the automorphisms $\alpha$ preserve $S, \alpha\left(w^{\prime}\right)$ is a word in $S$ of length $d$, from $H g^{\prime}$ to the trivial coset in the Schreier coset graph.

\section{Perfect hash function.}

Next, a perfect hash function is a hash function that produces no collisions. Hence it is one-to-one. Section 5 describes efficient perfect hash functions both for certain classes of symmetrized cosets and for symmetrized group elements. There is an efficiently computable perfect hash function for symmetrized cosets of our chosen subgroup of Rubik's group. While this perfect hash function is not minimal, it is nearly so. Furthermore, it has an efficiently computable inverse hash function.

\subsection{Rubik's cube definitions}

We assume the reader has seen a Rubik's cube, and we provide this description solely to fix terminology according to standard conventions [2].

A Rubik's cube is built from 26 cubies, each able to make restricted rotations about a core of Rubik's cube. A face of Rubik's cube is a side. Each face is divided into 9 facelets, where each of the 9 facelets is part of a distinct cubie. A cubie is either an edge cubie (two visible facelets), a corner cubie (three visible facelets), or a center cubie (one visible face, in the center of a side). The facelets are similarly edge facelets, corner facelets, or center facelets.

The states of Rubik's cube can be considered as permutations on 48 facelets (the 24 corner facelets and the 24 edge facelets). The center facelets are considered to be fixed, and all rotations of $\mathrm{Ru}-$ bik's cube are considered to preserve a fixed orientation of the cube in 3 dimensions. The home position or solved position of Rubik's cube is one in which all facelets of a face are the same color, and (for the sake of specificity) the blue face is downwards. We will speak interchangeably about an element of Rubik's group, a state, or a position of Rubik's cube. Similarly, we will speak interchangeably about the home position of Rubik's cube or the identity element of Rubik's group. Hence, a position of the cube is identified with a group element that permutes the facelets from the home position to the given position.

The moves of Rubik's group are conventionally denoted $U, U^{-1}$, $U^{2}, D, D^{-1}, D^{2}, R, R^{-1}, R^{2}, L, L^{-1}, L^{2}, F, F^{-1}, F^{2}, B, B^{-1}$, and $B^{2}$. Mnemonically, $U$ stands for a clockwise quarter turn of 
the "up" face, and similarly, $D, R, L, F$ and $B$ stand for "down", "right", "left", "front" and "back", respectively. Any of the above moves will move exactly 20 facelets. These moves also make up the generators of the Rubik's cube group, $G$. Standard techniques, such as Sims's algorithm for group membership, show that the order of Rubik's group is $|G|=43,252,003,274,489,856,000$ (approximately $4.3 \times 10^{19}$ ).

The generators of the square subgroup are given by

$$
Q=\left\langle U^{2}, D^{2}, R^{2}, L^{2}, F^{2}, B^{2}\right\rangle
$$

Standard techniques show that the order of the square subgroup is $|S|=663,552$ (approximately $6.6 \times 10^{5}$ ). The index, or number of cosets, is $[G: S]=|G| /|S|=65,182,537,728,000$ (approximately $6.5 \times 10^{13}$ ).

\subsection{Symmetries (Natural Automorphisms) of Rubik's Cube}

For Rubik's cube, we desire a subgroup of 48 automorphisms that preserve the set of generators: the natural automorphisms of Rubik's cube. Each automorphism can be identified with a symmetry of a geometric cube: either one of 24 rotations of the entire cube or a rotation followed by an inversion of the cube. An inversion of the cube maps each corner of the cube to the opposite corner.

A rotation of the cube maps the natural generators of Rubik's cube to generators. An inversion of the cube maps generators to inverse generators (clockwise quarter turns to counter-clockwise quarter turns). These 48 symmetries of the cube are known to preserve the natural generators of Rubik's group, and no other automorphisms of Rubik's group do so.

\section{FAST GROUP MULTIPLICATION IN THE PRESENCE OF SYMMETRIES}

Next, the method of fast multiplication is presented. The method breaks up a group into smaller subgroups (called coordinates by Kociemba [3]). The chosen subgroups are tailored for fast multiplication both within the square subgroup (group generated by squares of generators) and multiplication of the cosets by generators. Each subgroup is small enough so that the derived table-based computations fit inside CPU cache.

We will separately consider the edge group (the action on edge facelets) and the corner group (the action on corner facelets). The two actions are "linked". This will be discussed in later subsections. First, we provide the fundamental basis for our result.

\subsection{Decomposition into Smaller Subgroups and Fast Multiplication}

The following easy result provides the basis for our fast multiplication.

LEMMA 1. Let $G$ be a group with $G>H=Q N$ and $Q \cap N=$ $\{1\}$. Then given a set of canonical coset representatives of $G / H$, an element $g \in G$ uniquely defines $q, \bar{g}$ and $n$ as follows.

$$
\begin{array}{r}
g=q \bar{g} n, \text { where } q \in Q, \bar{g} \text { is the canonical coset } \\
\text { representative of } H g \text {, and } n \in N .
\end{array}
$$

Proof. Clearly, $g=h \bar{g}$ for a uniquely defined $h \in H$ and $\bar{g}$ the coset representative of $H g$. The equation $h=q n^{\prime}$ then uniquely defines $q$ and $n^{\prime}$ for $q \in Q$ and $n \in N$. Define $n^{\prime}$ by $n=n^{\prime \bar{g}}$. Then $g=h \bar{g}=q n^{\prime} \bar{g}=q \bar{g} n$.

Lemma 1 provides the basis for a perfect hash function for $G$ and $Q$, since $g$ and the corresponding coset $Q g$ can be encoded by $\phi_{1}$ and $\phi_{2}$ as follows.

$$
\begin{array}{rr}
\text { For } g=q \bar{g} n \text { as above }, & \phi_{1}(g)=(q, \bar{g}, n) \\
\text { and } & \phi_{2}(Q g)=(\bar{g}, n) .
\end{array}
$$

Given perfect hash functions for $Q, G / H$ and $N$, the above equations yield perfect hash functions for $G$ and for $G / Q$ through a mixed radix encoding. The importance of this particular encoding is that it adapts well to an algorithm for fast multiplication.

If the group $N$ is also normal, then there is an efficient multiplication of a triple $(q, \bar{g}, n)$ and a pair $(\bar{g}, n)$. Recall that the conjugate of a group element $h$ by $g$ is defined as $h^{g}=g^{-1} h g$. Recall that a subgroup $N<G$ is a normal subgroup if $\forall n \in N$, and $g \in G, n^{g} \in N$. This immediately implies $n g=g n^{g}$ and $n_{1}^{g} n_{2}^{g}=\left(n_{1} n_{2}\right)^{g}$ for $n, n_{1}, n_{2} \in N$ and $g \in G$.

Next, consider $g=q \bar{g} n$ as above. In addition, assume that $N$ is a normal subgroup. Let $\bar{g} s$ decompose into $q^{\prime} \overline{\bar{g} s} n^{\prime}$ by Lemma 1 , where $\overline{\bar{g} s}$ is the canonical coset representative of $H \bar{g} s$. Note also that $H \bar{g} s=H g s$ implies $\overline{\bar{g} s}=\overline{g s}$ for $\overline{g s}$ the canonical coset representative of $\mathrm{Hgs}$. Then the product of $g$ by a generator $s$ can be expressed as follows.

LEMMA 2. Let $Q<G$ and let $N$ be a normal subgroup of $G$. Let $Q \cap N=\{1\}$. Let $g, s \in G$. Assume the decompositions $g=$ $q \bar{g} n$ and $\bar{g} s=q^{\prime} \overline{g s} n^{\prime}$, as given by Lemma 1. Then the following holds.

$$
\begin{aligned}
\text { If } g=q \bar{g} n \text { and } \bar{g} s=q^{\prime} \overline{g s} n^{\prime}, \text { then } & g s=\left(q q^{\prime}\right) \overline{g s}\left(n^{\prime} n^{s}\right) \text { (3) } \\
\text { and } & Q g s=Q \overline{g s}\left(n^{\prime} n^{s}\right) . \text { (4) }
\end{aligned}
$$

Providing that $Q, N$ and $G /(Q N)$ are sufficiently small, Lemma 2 provides the foundation for precomputing small tables of all the required products, for any given $s \in G$. Typically, we choose $s$ to be a generator since there are few generators, and so the relatively small size of the tables is maintained.

Fast multiplication by an arbitrary group element, while using small tables, is also feasible. By Lemma 1, an arbitrary group element can be written as $g=q \bar{g} n$. Assuming that $g$ is represented as the triple $(q, \bar{g}, n)$, one can successively treat each of $q, \bar{g}$ and $n$ as the generator $s$ for purposes of multiplication on the right.

\subsection{Fast Multiplication of Coset by Generator for Edge Group}

First, consider the edge group $E$, the restriction of Rubik's group to act only on edge facelets. Similarly, let $M_{E}$ be the restriction of the generators of Rubik's cube, $M$, to elements that act only on the edges. Let $Q_{E}$ be the restriction of the square subgroup $Q=\left\langle\left\{s^{2} \mid s \in M\right\}\right\rangle$ to act only on the edges. Hence, $Q_{E}=$ $\left\langle\left\{s^{2} \mid s \in M_{E}\right\}\right\rangle$.

Let $N_{E}$ be the normal subgroup of $E$ that fixes the edge cubies setwise, but allows the two facelets of an edge cubie to be transposed. There are 12 edge cubies, but it is not possible in Rubik's cube to transpose the facelets of an odd number of edge cubies. Group theoretic arguments then show the order of the group $N_{E}$ to be $2^{11}$.

It is easy to show that $N_{E}$ is normal in $G$ since for $n \in N_{E}$ and $g \in G, g^{-1} n g$ may permute the cubies according to $g^{-1}$, but $n$ fixes the cubies, and $g$ then brings the cubies back to their original position. So, $g^{-1} n g \in N$ and so $N_{E}$ is normal.

We describe the method for fast multiplication of a coset of $E / Q_{E}$ by a generator from $M_{E}$. We define $H_{E}=Q_{E} N_{E}$. The method depends on a subgroup chain

$$
\begin{aligned}
E> & H_{E}>N_{E}, \\
& \text { for } N_{E} \text { normal in } E, \\
H_{E} \stackrel{\text { ef }}{=} Q_{E} N_{E}, & \text { and } Q_{E} \cap N_{E}=\{1\} .
\end{aligned}
$$

For the remainder of this section, we often omit the subscripts of $M_{E}, H_{E}, N_{E}$ and $Q_{E}$, since we will always be concerned with the action on edges.

By Lemma 1, for any coset $Q g \in E / Q, Q g=Q r_{1} r_{2}$. So, $Q g$ is represented as a pair $\left(r_{1}, r_{2}\right)$ for $r_{1}$ a canonical coset representative in $E / H$ and $r_{2} \in N$. Given a generator $s$ of $E$, equation 4 is 


\begin{tabular}{|l|l|rl|}
\hline Edge Tables & Size & Inputs & Output \\
\hline Table 1a & $1564 \times 18 \times 2 \mathrm{~B}$ & $r_{1}, s$ & $H \overline{r_{1} s} \in E / H$ for $\overline{r_{1} s}$ a canonical coset representative of $E / H$ \\
Table 1b & $1564 \times 18 \times 2 \mathrm{~B}$ & $r_{1}, s$ & $\bar{r}_{2} \stackrel{\text { def }}{=} n^{\overline{r_{1} s}} \in N$, where $n$ is defi ned by setting $h^{\text {def }}=\overline{r_{1} s}\left(r_{1} s\right)^{-1} \in H$ \\
& & and uniquely factoring $h=\bar{q} n$ for $\bar{q} \in Q, n \in N$ \\
Table 2 & $2048 \times 18 \times 2 \mathrm{~B}$ & $r_{2}, s$ & $r_{2}^{s} \in N$ \\
Logical op's & & $r_{2}, r_{2}^{\prime}$ & $r_{2} r_{2}^{\prime} \in N$ (using addition mod 2 on packed fi elds) \\
\hline
\end{tabular}

Figure 1: Edge table for fast multiplication

\begin{tabular}{|c|c|c|c|}
\hline Edge Tables & Size & Inputs & Output \\
\hline Table Aut & $1564 \times 18 \times 1 \mathrm{~B}$ & $r_{1, e}, s$ & $\begin{array}{l}\alpha \in A \text { for } \overline{\alpha\left(r_{1} s\right)} \text { a canonical coset rep. of } H \text { in } E \\
\text { (We choose } \alpha \text { such that } \overline{\alpha\left(r_{1} s\right)}=\min _{\beta \in A} \overline{\beta\left(r_{1} s\right)} \text { ) }\end{array}$ \\
\hline $\begin{array}{l}\text { Table 1a } \\
\text { (coset rep.) }\end{array}$ & $1564 \times 18 \times 2 B$ & $r_{1, e}$ & $\begin{array}{l}H \overline{\alpha\left(r_{1} s\right)} \in E / H \text { for } \alpha \text { defi ned in terms of } r_{1} \text { and } s \text { by Table Aut. } \\
\text { (Note that } H^{A}=H \text {.) }\end{array}$ \\
\hline $\begin{array}{l}\text { Table } 1 \mathrm{~b} \\
(N)\end{array}$ & $1564 \times 18 \times 2 B$ & $r_{1, e}, s$ & $\begin{array}{l}\bar{r}_{2} \stackrel{\text { def }}{=} n^{\prime \overline{\alpha\left(r_{1} s\right)}} \in N, \text { where } h^{\prime} \stackrel{\text { def }}{=} \alpha\left(r_{1} s\right) \overline{\alpha\left(r_{1} s\right)}-1 \in H \text { and } h^{\prime}=\bar{q}^{\prime} n^{\prime} \\
\text { for } \bar{q}^{\prime} \in Q, n^{\prime} \in N \text { for } \alpha \text { defi ned in terms of } r_{1} \text { and } s \text { by Table Aut }\end{array}$ \\
\hline Table 2 & 2048 & $r_{2, e}, s$ & $r_{2}^{s} \in N$ \\
\hline Table 5 & $2048 \times 48 \times 2 B$ & & $\begin{array}{l}\alpha(n) \in N, \text { where } \alpha \text { is the output of Table Aut } \\
n \text { is defi ned by } n=r_{2}^{s} \text { (output of Table } 2 \text { for edges) }\end{array}$ \\
\hline Logical op's & & $r_{2, e}, r_{2, e}^{\prime}$ & $r_{2} r_{2}^{\prime} \in N$ (using addition mod 2 on packed fi elds) \\
\hline
\end{tabular}

Figure 2: Edge table for fast multiplication of symmetrized coset by generator

used below to multiply the pair $\left(r_{1}, r_{2}\right)$ by $s$ and return a new pair, $\left(r_{1}^{\prime}, r_{2}^{\prime}\right)=\left(\overline{r_{1} s}, \bar{r}_{2}\left(r_{2}^{s}\right)\right)$.

$$
\begin{aligned}
& \text { Let } r_{1} s=\bar{q} \overline{r_{1} s} \bar{r}_{2}, \\
& \text { where } \bar{q} \in Q, \bar{r}_{2} \in N, \text { and } \overline{r_{1} s} \text { is the } \\
& \text { canonical coset representative of } Q r_{1} s .
\end{aligned}
$$

Given $\left(r_{1}, r_{2}\right)$ and a generator $s$, one can compute $\left(r_{1}^{\prime}, r_{2}^{\prime}\right)$ such that $Q r_{1} r_{2} s=Q r_{1}^{\prime} r_{2}^{\prime}$ primarily through table lookup. Figure 1 describes the necessary edge tables.

Note that the logical operations can be done efficiently, because $N$ is an elementary abelian 2-group. This means that the group $N$ is isomorphic to an additive group of vectors over a finite field of order 2. In other words, multiplication in $N$ is equivalent to addition in $\operatorname{GF}(2)^{11}$, the 11-dimensional vector space over the field of order 2. Addition in the field of order 2 can be executed by "exclusive or". Hence, it suffices to use bitwise "exclusive or" over 11 bits for group multiplication in $N_{E}$.

\subsection{Extension to Group Action on Corners}

For corners, we use the same logic as previously, but with the corner group $C$, the restriction $M_{C}$ of the generators to corners, and the restriction $Q_{C}$ of the square group to corners. Let $N_{C}$ be the subgroup of $C$ that fixes in position the corner cubies, but allows the facelets of the corner cubie to be permuted. There are 8 corner cubies, but a standard group-theoretic algorithm shows that within the subgroup $C$, the number of group elements fixing all corner cubies is only $3^{7}$.

As before, we drop the subscripts for readability. Hence, the tables of Figure 1 can be reinterpreted as pertaining to corners. However, there is a small difference. Since $N_{C}$ is an elementary abelian 3-group, multiplication in $N_{C}$ is equivalent to addition over $\operatorname{GF}(3)^{7}$.

\subsection{Generalization to Fast Multiplication over Symmetrized Cosets}

Assume that the automorphism group $A$ acts on edge facelets and corner facelets, separately preserving edge and corner facelets. Assume also that $A$ preserves the subgroups $Q, N$ and $H=Q N$. Therefore, $A$ also maps the projections of $Q, N$ and $H=Q N$ into edges and into corners.

Assume that the symmetrized $\operatorname{coset} Q g^{A}$ is uniquely represented as $Q\left(r_{1} r_{2}\right)^{A}$, where $r_{1}$ is the canonical representative of a symmetrized coset $\mathrm{Hg}^{A}$ with $H=Q N$, and $r_{2} \in N$, as described in Lemma 1.

The subscript $e$, below, indicates the restriction of a permutation to its action only on edges. Similarly, the subscript $c$ is for corners. The subscripts are omitted where the meaning is clear.

For edges,

$Q \alpha\left(r_{1, e} r_{2, e} s\right)=Q \alpha\left(r_{1} s\right) \alpha\left(r_{2}^{s}\right)=Q \overline{\alpha\left(r_{1} s\right)}\left(\bar{r}_{2} \alpha\left(r_{2}^{s}\right)\right)$, where $\overline{\alpha\left(r_{1} s\right)}$ defined by Table 1 a for edges,

where $\alpha$ is chosen to minimize $\overline{\alpha\left(r_{1} s\right)}$,

$\bar{r}_{2}$ defined by Table $1 \mathrm{~b}$ for edges, etc.

However for corners,

$Q \alpha\left(r_{1, c} r_{2, c} s\right)=Q \alpha\left(\overline{r_{1} s}\left(\bar{r}_{2}\left(r_{2}^{s}\right)\right)\right)=$
$Q \alpha\left(\overline{r_{1} s}\right) \alpha\left(\bar{r}_{2}\left(r_{2}^{s}\right)\right)=Q \overline{\alpha\left(\overline{r_{1} s}\right)} n^{\overline{\alpha\left(\overline{r_{1}}\right)}} \alpha\left(\bar{r}_{2}\left(r_{2}^{s}\right)\right)$,

where $\alpha$ is chosen as in equation 7,

where $\overline{r_{1} s}$ defined by Table 1a,

$\overline{\alpha\left(\overline{r_{1} s}\right)}$ defined by Table $4 \mathrm{a}$,

$n^{\overline{\alpha\left(\overline{r_{1} s}\right)}}$ defined by Table $4 \mathrm{~b}$ for corners,

$\bar{r}_{2}$ defined by Table $1 \mathrm{~b}$,

$r_{2}^{S}$ by Table 2 for corners, etc.

Note that the choice of $\alpha \in A$ for edges above depends on there being a unique such automorphism that minimizes $\overline{\alpha\left(r_{1} s\right)}$. In fact, this is not true in about $5.2 \%$ of cases for randomly chosen $r_{1}$ and $s$. These unusual cases can be easily detected at run-time, and additional tie-breaking logic is generated. We proceed to describe tables for fast multiplication for the common case of unique $\alpha \in A$ minimizing $\overline{\alpha\left(r_{1} s\right)}$, and discuss the tie-breaking logic later.

The tables that implement the above formulas follow. While it is mathematically true that we can simplify $\overline{\alpha\left(\overline{r_{1} s}\right)}$ into $\overline{\alpha\left(r_{1} s\right)}$, we often maintain the longer formula to make clear the origins of that expression, which is needed for an implementation. As before, the subscripts $e$ and $c$ indicate the restriction of a permutation to its action only on edges and only on corners. Figures 2 and 3 describe the following edge tables, among others.

Ideally, one would use only the simpler formula and tables for edges, and copy that logic for corners. Unfortunately, this is not 


\begin{tabular}{|c|c|c|}
\hline Corner Tables & Size & Output \\
\hline Table 1a & $420 \times 18 \times 2 \mathrm{~B}$ & $H \overline{r_{1} s} \in C / H$ for $\overline{r_{1} s}$ a canonical rep. of a coset of $C / H$ \\
\hline Table $1 \mathrm{~b}$ & $420 \times 18 \times 2 B$ & $\begin{array}{l}\bar{r}_{2} \stackrel{\text { def }}{=} n^{\prime \overline{r_{1}} s} \in N \text {, where } n^{\prime} \text { is defi ned by setting } h^{\text {def }}=r_{1} s \overline{r_{1} s}-1 \\
\text { and uniquely factoring } h=\bar{q} n^{\prime} \text { for } \bar{q} \in Q, n^{\prime} \in N\end{array}$ \\
\hline Table 2 & $2187 \times 18 \times 2 \mathrm{~B}$ & $r_{2}^{s} \in N$ \\
\hline $\begin{array}{l}\text { Table } 4 \mathrm{a} \\
\text { (coset rep.) }\end{array}$ & $420 \times 48 \times 2 B$ & $\begin{array}{c}H \overline{r_{1, c} s} \in C / H, \alpha \quad H \overline{\alpha\left(\overline{r_{1} s}\right)} \in C / H, \text { where } H \overline{r_{1} s} \text { is the output of Table 1a, } \\
\text { and } \alpha \text { is the output of Table Aut on edges }\end{array}$ \\
\hline $\begin{array}{l}\text { Table } 4 \mathrm{~b} \\
(N)\end{array}$ & $420 \times 48 \times 2 B$ & $\begin{aligned} H \overline{r_{1, c} s} \in C / H, \alpha \quad n^{\overline{\alpha\left(\overline{r_{1} s}\right)}} \in N, \text { where } H \overline{r_{1} s} \text { is the output of Table } 1 \mathrm{a}, \\
\text { and } n \text { is defi ned by setting } h=\alpha\left(\overline{r_{1} s}\right) \overline{\alpha\left(\overline{r_{1} s}\right)}-1 \in H, \\
\text { and uniquely factoring } h \text { into } q n \text { for } q \in Q, n \in N\end{aligned}$ \\
\hline Logical op's & $2187 \times 48 \times 2 B$ & $\begin{aligned} n_{c} \in N, \alpha \quad & \alpha(n) \in N, \text { where } \alpha \text { is the output of Table Aut on edges } \\
& n \text { defi ned by computing } \bar{r}_{2}=n^{\prime r_{1}^{s}} \text { (as in Table 1b), } \\
& \text { and } r_{2}^{s} \text { computed as in Table } 2, \\
& \text { and } \bar{r}_{2} r_{2}^{s} \text { computed by logical op's on corners } \\
r_{2, c}, r_{2, c}^{\prime} & r_{2} r_{2}^{\prime} \in N \text { (using addition mod } 3 \text { on packed fi elds) }\end{aligned}$ \\
\hline
\end{tabular}

Figure 3: Corner table for fast multiplication of symmetrized coset by generator

possible. We must choose a representative automorphism $\alpha \in A$ for purposes of computation. We choose $\alpha$ based on the projection $r_{1, e}$ of $r_{1}$ into $E$ (action of $r_{1}$ on edges). Hence, Tables 1a and $1 \mathrm{~b}$ for edges take input $r_{1}$ and $s$, then compute $\alpha$ as an intermediate computation, then return $H \overline{\alpha\left(r_{1} s\right)}$. A similar computation for corners is not possible, because the intermediate value $\alpha$ depends on $r_{1, e}$ and not on the corresponding element of the corner group $r_{1, c}$.

Tie-breakers: when the minimizing automorphism is not unique.

Table Aut in the previous table for edges defines an automorphism $\alpha$ that minimizes $\overline{\alpha\left(r_{1} s\right)}$. Unfortunately, there is not always a unique such $\alpha$. In such cases, one needs a tie-breaker, since different choices of $\alpha$ will in general produce different encodings (different hash indices).

For each possible value of $\overline{\alpha\left(r_{1} s\right)}$, with $\alpha$ chosen to minimize the expression, we precompute the stabilizer subgroup $B \leq A$ defined by

$$
B=\left\{\beta \in A: \overline{\left.\overline{\beta\left(\alpha\left(r_{1} s\right)\right.}\right)}=\overline{\alpha\left(r_{1} s\right)}\right\}
$$

we use the formulas and additional table below to find the unique $\beta \in B$ such that the product $\alpha \beta$ minimizes the edge pair result $\left(r_{1, e}^{\prime}, r_{2, e}^{\prime}\right)$. Where even this is not enough to break ties, we compute the full encoding, while trying all possible tying automorphisms. This latter situation arises in only $0.23 \%$ of the time, and does not contribute significantly to the time. The tables of Figure 4 suffice for these computations.

For edges,

$$
\begin{aligned}
& Q \beta\left(\alpha\left(r_{1, e} r_{2, e} s\right)\right)=Q \beta\left(\alpha\left(r_{1} s\right)\right) \beta\left(\alpha\left(r_{2}^{s}\right)\right)= \\
& Q \beta\left(\overline{\alpha\left(r_{1} s\right)}\right)\left(\beta\left(\bar{r}_{2} \alpha\left(r_{2}^{s}\right)\right)\right)=Q \overline{\alpha\left(r_{1} s\right)} \bar{r}_{2}^{\prime}\left(\beta\left(\bar{r}_{2} \alpha\left(r_{2}^{s}\right)\right)\right), \\
& \text { where } \overline{\alpha\left(r_{1} s\right)} \text { defined by Table 1a for edges, } \\
& \text { where } \alpha \text { is chosen to minimize } \overline{\alpha\left(r_{1} s\right)}, \\
& \text { and } \beta \in A \text { satisfies } Q \overline{\beta\left(\overline{\alpha\left(r_{1} s\right)}\right)}=Q \overline{\alpha\left(r_{1} s\right)} \\
& \text { and } \beta\left(\overline{\alpha\left(r_{1} s\right)}\right)=\overline{\alpha\left(r_{1} s\right)} \bar{r}_{2}^{\prime}\left(r_{2}^{\prime}\right. \text { defined in Table 3), } \\
& \text { and } \bar{r}_{2} \text { defined by Table } 1 \mathrm{~b} \text { for edges. }
\end{aligned}
$$

However for corners,

$$
\begin{aligned}
& Q \beta\left(\alpha\left(r_{1, c} r_{2, c} s\right)\right)=Q \beta\left(\alpha\left(\overline{r_{1} s}\left(\bar{r}_{2}\left(r_{2}^{s}\right)\right)\right)\right)= \\
& Q \beta\left(\alpha\left(\overline{r_{1} s}\right)\right) \beta\left(\alpha\left(\bar{r}_{2}\left(r_{2}^{s}\right)\right)\right)= \\
& Q \overline{\beta\left(\alpha\left(\overline{r_{1} s}\right)\right)} n^{\overline{\beta\left(\alpha\left(\overline{r_{1} s}\right)\right)}} \beta\left(\alpha\left(\bar{r}_{2}\left(r_{2}^{s}\right)\right)\right) \\
& \text { where } \alpha \text { and } \beta \text { are chosen as in equation } 9, \\
& \text { and other quantities based on } \\
& \text { the previous Corner Tables using } \alpha \beta .
\end{aligned}
$$

Table $1 \mathrm{c}$ is implemented more efficiently by storing the elements of each of the possible 98 subgroups of the automorphism group, and having Table $1 \mathrm{c}$ point to the appropriate subgroup $B \leq A$, stabilizing $r_{1, e}, s$.

\subsection{Optimizations}

In the discussion so far, we produce the encoding or hash index of a group element based on an encoding of the action of the group element on edges, along with an encoding of the action of the group element on corners. We can cut this encoding in half due to parity considerations.

Consider the action of Rubik's cube on the 12 edge cubies and the 8 corner cubies, rather than on the facelets. We define the edge parity of a group element to be the parity (even or odd) in its action on edge cubies. (Recall that the parity of a permutation is odd or even according to whether the permutation is expressible as an odd or even number of transpositions.) The corner parity is similarly defined.

The edge and corner parity of a symmetrized coset, $\mathrm{Hg}^{A}$, are well-defined, and are the same as the edge and corner parity of $g$. This is so because $H=Q N$, and elements of $Q$ and $N$ have even edge parity and even corner parity. Parity is unchanged by the action of an automorphism.

For Rubik's cube, the natural generators have the edge parity equal to the corner parity. So this property extends to all group elements, and hence to all symmetrized cosets $\mathrm{Hg}^{A}$. Therefore, our encoding can assume that edge and corner parities of symmetrized cosets are equal. The size of the corresponding hash table is thus reduced by half.

\section{Nearly Minimal Perfect Hash Function.}

If we were to only use cosets instead of symmetrized cosets (no automorphism), then the perfect hash function that we have described implicitly would also be a minimal perfect hash function. However, there are examples for which $Q \alpha(g)=Q g$ for $\alpha$ not the identity automorphism.

A computation demonstrates that the perfect hash function of Section 5.4 with the addition of the parity optimization has an efficiency of $92.3 \%$. In fact, we compute that there are

$$
1,471,074,877,440 \approx 1.5 \times 10^{12} \approx|G| /|Q| / 44.3
$$

symmetrized cosets. The ratio $44.3 / 48$ yields our efficiency ratio of $92.3 \%$. Further details are omitted due to lack of space.

\subsection{Fast Multiplication in Square Subgroup}

There is a similar algorithm for fast multiplication in the square subgroup, which is omitted due to lack of space. 


\begin{tabular}{|c|c|c|}
\hline Edge Tables & Size & Output \\
\hline Table Mult Aut & $48 \times 48 \times 1 \mathrm{~B}$ & $\alpha, \beta \quad$ the product $\alpha \beta \in A$ \\
\hline Table 1c $(A)$ & $1564 \times 18 \times 1 B$ & $r_{1, e}, s \quad\left\{\beta \in A: \overline{\beta\left(\overline{\alpha\left(r_{1} s\right)}\right)}=\overline{\alpha\left(r_{1} s\right)}\right\}$ \\
\hline Table $3(N)$ & $2048 \times 48 \times 2 B$ & $\begin{aligned} H \overline{r_{1, c} s} \in C / H, \alpha \quad \bar{r}_{2}^{\prime} \stackrel{\text { def }}{=} n^{\prime \prime} \overline{\beta\left(r_{1}\right)} \in N, \text { where } \beta \text { taken from Table 1c, and } \\
\\
\text { where } h^{\prime \prime} \stackrel{\text { def }}{=} \beta\left(r_{1}\right) \overline{\beta\left(r_{1}\right)}-1 \in H, \text { and } h^{\prime \prime}=\bar{q}^{\prime \prime} n^{\prime \prime}, \text { for } \bar{q}^{\prime \prime} \in Q, n^{\prime \prime} \in N\end{aligned}$ \\
\hline
\end{tabular}

Figure 4: Edge table for fast multiplication of symmetrized coset by generator, adjusted to break ties

\section{6. "BRUTE FORCE" UPPER BOUNDS ON SOLUTIONS WITHIN A COSET}

\subsection{Goal of Brute Forcing Cosets}

Having constructed the Schreier coset graph, one wishes to test individual cosets, and prove that all group elements of that coset are expressible as words in the generators of length at most $u$. Hence, $u$ is the desired upper bound we wish to prove.

Recall that $G$ is the group of Rubik's cube, and $Q$ is the square subgroup. Consider a coset $Q g$ at a level $\ell$ (distance $\ell$ from the home position, or identity coset, in the coset graph). Let $d$ be the diameter of the subgroup $Q$. For any group element $h \in Q g$, clearly its distance from the identity element is at least $\ell$ and at most $\ell+d$. We describe a computation to produce a finer upper bound on the distance of any $h \in Q g$ from the identity element.

Because our subgroup $Q$ (the square subgroup) is of such small order, it is even feasible to simply apply an optimal solver to each element of a coset. If the optimal length words for each element is always less than or equal to $u$, then we are done. However, we will present a more efficient technique, which can scale to millions of cosets.

For simplicity, we first assume that we are not applying any symmetry reductions using the automorphism group. So, each coset contains 663,552 elements, just as does the square subgroup.

\subsection{Basic Algorithm}

Note that for the coset $Q g$, there can be many paths in the coset graph from the identity coset to $Q g$. In terms of group theory, there are multiple words, $w_{1}, w_{2}, \ldots$, where each word is a product of generators of Rubik's group, and $Q w_{1}=Q w_{2}=\ldots=Q g$. Note that in general, the words are distinct group elements: $w_{1} \neq w_{2}$, etc. Nevertheless, $w_{1} w_{2}^{-1} \in Q$. This is the key to finding a refined upper bound.

Next, suppose our goal is to demonstrate an upper bound $u$, for $\ell \leq u<\ell+d$. Let $\operatorname{dist}(q)$ denote the distance from an element $q \in Q$ to the identity element in the Cayley graph of $G$ with the original Rubik generators. Let $Q_{u}$ be $\{q \in Q \mid \operatorname{dist}(q) \leq u\}$, the subset of $Q$ at distance from the identity at most $u$.

Next, consider $Q_{k} g \stackrel{\text { def }}{=}\left\{q g \mid q \in Q_{k}\right\}$. Assume that the words $w_{i}$ are of length $d$ in the generators of Rubik's group, and that $Q w_{1}=Q w_{2}=\ldots=Q g$. Note that for all elements of $Q_{k} w_{1}$, there is an upper bound, $k+d$. Similarly, for all elements of $Q_{k} w_{2}$, there is an upper bound, $k+d$. Therefore, the elements of $Q_{k} w_{1} \cup Q_{k} w_{1}$ have an upper bound of $k+d$. More compactly,

$$
\operatorname{dist}\left(Q_{k} w_{1} \cup Q_{k} w_{2}\right) \leq k+d
$$

More generally, for $w_{i}$ a word in the generators of $G$, let len $\left(w_{i}\right)$ be the length of that word. Then

$$
\operatorname{dist}\left(Q_{k+d-\operatorname{len}\left(w_{1}\right)} w_{1} \cup Q_{k+d-\operatorname{len}\left(w_{2}\right)} w_{2}\right) \leq k+d
$$

since the length of any word in $Q_{k+d-\operatorname{len}\left(w_{1}\right)} w_{1}$ is at most $(k+$ $\left.d-\operatorname{len}\left(w_{1}\right)\right)+\operatorname{len}\left(w_{1}\right)=k+d$ and similarly for $w_{2}$.

Define the complement $Q_{j}^{\prime} \stackrel{\text { def }}{=} Q \backslash Q_{j}$. We can now write:

$Q_{k+d-\operatorname{len}\left(w_{1}\right)}^{\prime} w_{1} \cap Q_{k+d-\operatorname{len}\left(w_{2}\right)}^{\prime} w_{2} \supseteq\{h \in Q g \mid \operatorname{dist}(h g)>k+d\}$
Clearly, the above equation can be generalized to the intersection of multiple words, $w_{1}, w_{2}, \ldots$.

$\cap_{i} Q_{k+d-\operatorname{len}\left(w_{i}\right)}^{\prime} w_{i}=\emptyset \Longrightarrow \forall i, \operatorname{dist}\left(Q w_{i}\right)=\operatorname{dist}(Q g) \leq k+d$

Finally, for purposes of computation, Algorithm 2, below, captures these insights.

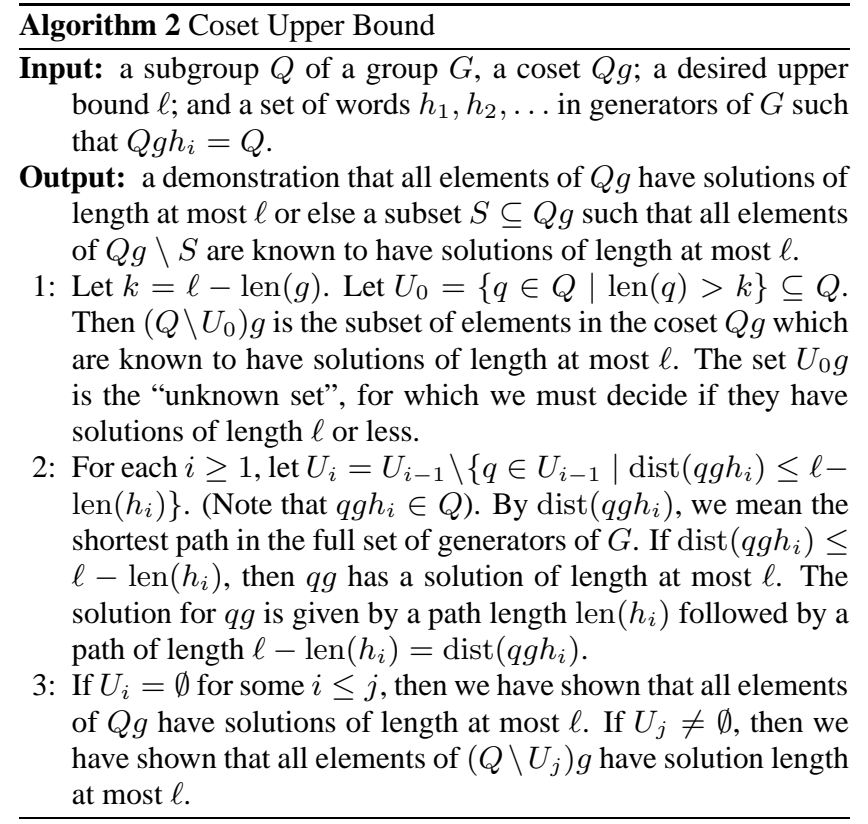

For purposes of implementation, note that $g h_{i} \in Q$. So, for $q \in Q, q g h_{i}$ can be computed by fast multiplication within the subgroup $Q$.

\subsection{Using Symmetries}

We now generalize the method of the previous section to take account of reductions through symmetries.

First, note that for a symmetrized coset with representative coset $Q g$ and for a natural automorphism $\alpha$, $\operatorname{dist}(Q g)=\operatorname{dist}(Q \alpha(g))$. (This was demonstrated in Section 3.2). Furthermore, for any $h \in Q g$, $\operatorname{dist}(h)=\operatorname{dist}(\alpha(h))$.

From this, it is clear that any upper bound on the distance of elements in $Q g$ from the identity will also hold for $Q \alpha(g)$. So, it suffices to determine upper bounds for a single representative of each automorphism class.

Finally, one must determine whether $h w_{i}^{-1} \notin Q_{k+d-\operatorname{len}\left(w_{i}\right)}^{\prime}$. This can be done by maintaining a hash table mapping all elements $q \in Q$ to $\operatorname{dist}(q)$.

\section{EXPERIMENTAL RESULTS}

Our experimental results have proven that 26 moves suffice for any state of Rubik's cube. This was achieved in three steps: proving that all elements of the square subgroup are within 13 of the identity; proving that all cosets are within 16 of the trivial coset; and, 
refining the bound on the farthest cosets by brute force, reducing the bound by 3 .

\subsection{Square Subgroup Elements are within 13 of the Identity}

Recall, from Section 3.1, the following two-step process for this computation. First, we constructed the Cayley graph of the subgroup by breadth-first search, using the square generators. Then, the distance for each of these elements from the identity, when allowing all generators of the full group, were determined using bidirectional search. All of these computations were done on a single computer in under a day.

Table 1 shows the distribution of element distances in the square subgroup, using either the square generators or the full set of generators.

\begin{tabular}{|c|c|c|c|c|c|c|c|}
\hline \multicolumn{4}{|c|}{ Square Generators } & \multicolumn{4}{|c|}{ All Generators } \\
\hline Dist. & Elts & Dist. & Elts & Dist. & Elts & Dist. & Elts \\
\hline 0 & 1 & 8 & 1258 & 0 & 1 & 8 & 1871 \\
\hline 1 & 1 & 9 & 2627 & 1 & 1 & 9 & 4093 \\
\hline 2 & 2 & 10 & 4094 & 2 & 2 & 10 & 5394 \\
\hline 3 & 5 & 11 & 4137 & 3 & 5 & 11 & 2774 \\
\hline 4 & 18 & 12 & 2231 & 4 & 18 & 12 & 620 \\
\hline 5 & 56 & 13 & 548 & 5 & 62 & 13 & 4 \\
\hline 6 & 162 & 14 & 114 & 6 & 214 & & \\
\hline 7 & 482 & 15 & 16 & 7 & 693 & & \\
\hline & & Total & 15752 & & & Total & 15752 \\
\hline
\end{tabular}

Table 1: Distribution of elements in the square subgroup, after reduction by symmetries.

\subsection{Cosets are within 16 of the Trivial Coset}

The dominant time for our computations was in producing the symmetrized Schreier coset graph for the square subgroup in the group of Rubik's cube, as described in Section 3.2.

The computation used the DataStar cluster at the San Diego Supercomputer Center (SDSC). We used 16 compute nodes in parallel, each with 8 CPUs and 16 GB of main memory. For outof-core storage, we used DataStar's attached GPFS (IBM General Parallel File System). We used up to 7 terabytes of storage at any given time, as a buffer for newly generated states in the breadthfirst search. The final data structure, associating a 4-bit value with each symmetrized coset, used approximately 685 GB.

The computation required 63 hours, or over 8000 CPU hours. The fast multiplication algorithm allowed us to multiply a symmetrized coset by a generator at a rate between 5 and 10 million times per second, depending on the size of available CPU caches.

Table 2 shows the distribution of distances for cosets in the symmetrized Schreier coset graph.

\begin{tabular}{|r|r||r|rl|}
\hline Dist. & Elements & Distance & Elements & \\
\hline 0 & 1 & 9 & 80741117 & $\approx 8.1 \times 10^{7}$ \\
1 & 1 & 10 & 1028869318 & $\approx 1.0 \times 10^{9}$ \\
2 & 3 & 11 & 12787176355 & $\approx 1.3 \times 10^{10}$ \\
3 & 23 & 12 & 140352357299 & $\approx 1.4 \times 10^{11}$ \\
4 & 241 & 13 & 781415318341 & $\approx 7.8 \times 10^{11}$ \\
5 & 3002 & 14 & 421980213679 & $\approx 4.2 \times 10^{11}$ \\
6 & 38336 & 15 & 330036864 & $\approx 3.3 \times 10^{8}$ \\
7 & 490879 & 16 & 17 & \\
8 & 6298864 & & & \\
\hline & & Total & 1357981544340 & $\approx 1.36 \times 10^{12}$ \\
\hline
\end{tabular}

Table 2: Distribution of symmetrized cosets of the square subgroup.

\subsection{Brute Forcing of 3 Levels}

Kociemba's Cube Explorer software [3] was used to show that all cosets at level 3 have solutions of length at most 14 . To do this, it sufficed to analyze the elements at levels 12 and 13 from the square subgroup. Denoting the set of those subgroup elements $S$, and denoting all of the elements at level 3 in the cosets by $T$, we considered all pairwise products $S \times T$. There are $(620+4) \times$ $23=14,352$ such elements. Cube Explorer was run on each such element. This proves that for a coset at coset level $x>3,(x-$ $2)+13$ moves suffice. Combining this with the expected depth of $x=16$ for the symmetrized Schreier coset graph yields an upper bound of $(x-2)+13 \leq 27$ moves for solutions to Rubik's cube.

We similarly showed that none of the elements in any of the 17 cosets at level 16 required more than 26 moves, again using Cube Explorer. In combination with the above, this demonstrates an upper bound of 26 moves for solutions to Rubik's cube.

\subsection{Further Brute Forcing}

Our continuing work is using the efficient brute forcing techniques given in Section 6 to further reduce the upper bound from 26 to 25 moves. We plan to achieve this by brute forcing all cosets at some early level by three moves.

Our current experiments indicate that there exist elements that can not be brute forced by three moves out to level 8. Directly considering all cases at level 9 is computationally expensive, as there are over 80 million level 9 cosets, each with 3398 unproven elements (corresponding to the last three levels of the square subgroup). Instead, we use the new brute forcing techniques for cosets at earlier levels, and "project" the remaining unproven elements to later levels.

So far, we have removed over $95 \%$ of the elements across all cosets at level 8 . However, only about $10 \%$ of the cosets at level 8 have no remaining cases, and require additional brute forcing. We anticipate that, by using our efficient brute forcing technique, and significant computing power, we will be able handle the remaining cases at levels 8 and 9 , and therefore prove that 25 moves suffice for Rubik's cube.

\section{REFERENCES}

[1] Gene Cooperman, Larry Finkelstein, and Namita Sarawagi. Applications of Cayley graphs. In AAECC: Applied Algebra, Algebraic Algorithms and Error-Correcting Codes, International Conference, pages 367-378. LNCS, Springer-Verlag, 1990.

[2] Alexander H. Frey, Jr. and David Singmaster. Handbook of Cubik Math. Enslow Publishers, 1982.

[3] Herbert Kociemba. Cube Explorer. http: //kociemba.org/cube.htm, 2006.

[4] Richard Korf. Finding optimal solutions to Rubik's cube using pattern databases. In Proceedings of the Workshop on Computer Games (W31) at IJCAI-97, pages 21-26, Nagoya, Japan, 1997.

[5] Silviu Radu. Rubik can be solved in $27 f$. http: //cubezzz . homelinux.org/drupal/ ?q= node/view/53, 2006.

[6] Michael Reid. New upper bounds. http: //www. math. rwth-aachen. de/ Martin. Schoenert/Cube-Lovers/michael_reoid_new_ upper_bounds.html, 1995.

[7] Michael Reid. Superflip requires 20 face turns. http: //www. math. rwth-aachen. de/ Martin. Schoenert/Cube-Lovers/michael_re\%id_ _superflip_requires_20_face_turns.html, 1995 . 